\title{
Modelación y simulación computacional de fluido del Piptocoma discolor (pigüe) para la optimización de la combustión en el Ecuador
}

\section{(Modeling and computational simulation of Piptocoma discolor (Pigüe) fluid for combustión optimization in Ecuador)}

\author{
Juan Elías González ${ }^{1}$, Deny Oliva M. ${ }^{2}$, David Zambrano Vera ${ }^{3}$, Rubén Darío Ledesma4, \\ Mario Jorge Bonilla ${ }^{5}$, Erika Clara Casco G. ${ }^{6}$, Édison Ernesto Zúñiga ${ }^{7}$
}

\begin{abstract}
Resumen
La modelación y simulación computacional representan una herramienta competitiva y funcional que apoya eficazmente al estudio del comportamiento termoquímico en la optimización de la combustión. Garantiza resultados confiables a través de la modelación y diseño, y permitió la validación de las propiedades termodinámicas en exceso aplicados para la combustión de la biomasa del Piptocoma discolor (pigüe) de la región amazónica del Ecuador y América Latina. Los parámetros del modelo fueron físicos, químicos y térmicos a través de los fluidos dinámicos computacionales en optimización de la combustión. Se obtuvo el modelo matemático de la combustión bajo el método de transporte de la especie. En la simulación se consideró las variables del flujo de aire primario, contenido de humedad. Las variables de los parámetros de la combustión fueron N2, 02, C0, H, presión, temperatura, humedad, energía total y tiempo de quemado de las partículas de biomasa. La configuración geométrica fue desarrollada según los principios fundamentales de CFD dentro de Ansys Fluent para obtener el comportamiento de la velocidad, turbulencia, presión, etc. de la composición del combustible óptimo 91.75 \% de una combustión completa y frente a otras especies que arrojan el 86 \% de las reacciones de la combustión de las especies.
\end{abstract}

\section{Palabras clave}

Piptocoma discolor, modelación, simulación, optimización, combustión

\begin{abstract}
Computational modeling and simulation represents a competitive and functional tool that effectively supports the study of thermochemical behavior in combustion optimization, guaranteeing reliable results through modeling, design and allowed the validation of excess thermodynamic properties applied for combustion. of the biomass of Piptocoma discolor (pigüe) from the Amazonian region of Ecuador and Latin America. The model parameters were physical, chemical and thermal through computational fluid dynamics in combustion optimization. And the mathematical model of combustion was obtained under the transport method of the species. In the simulation, the variables of primary air flow and moisture content were observed. The variable of the combustion parameters were N2, 02, CO, $H$, pressure, temperature, humidity, total energy and burning time of the biomass particles. The geometric configuration was developed according to the fundamental principles of CFD within Ansys Fluent to obtain the behavior of speed, turbulence, pressure, etc. of the composition of the optimal fuel $91.75 \%$ of a complete combustion and compared to other species that show $86 \%$ of the reactions of the combustion of the species.
\end{abstract}

\section{Keywords}

Piptocoma discolor, modeling, simulation, optimization, combustion

1 Universidad Estatal Amazónica. Pastaza, Ecuador. [jgonzalez@uea.edu.ec, https://orcid.org/0000-0002-0674-7741]

2 Universidad Tecnológica de la Habana José Antonio Echeverría (Cujae). Habana, Cuba. [deny@aceter.cujae.edu.cu, https:// orcid.org/0000-0001-8117-3605]

3 Universidad Estatal Amazónica. Pastaza, Ecuador. [dzambrano@uea.edu.ec, https://orcid.org/0000-0002-2121-0205]

4 Universidad Estatal Amazónica. Pastaza, Ecuador. [rledesma@uea.edu.ec, https://orcid.org/0000-0002-2086-0185]

5 Consultor ambiental, registro MAE-SUIA-0249-Cl. Pastaza, Ecuador. [oncejorge11@yahoo.es, https://orcid.org/0000-0002-1328-5602]

6 Corporación de Desarrollo Integral Rashellbella (CORDEIR). Bolívar, Ecuador. [claracasco369agmail.com, https://orcid. org/0000-0002-6603-6837]

$7 \quad$ Unidad Educativa Intercultural Bilingüe Canelos. Pastaza, Ecuador. [ezunigalopez12@gmail.com, https://orcid.org/00000002-0605-7978] 


\section{Introducción}

El desarrollo de los sistemas CAD avanzado ha permitido desarrollar los campos de aplicación de la dinámica de los fluidos computacionales (CFD). Este método constituye una de las ramas de la ingeniería mecánica que utiliza los métodos numéricos y algoritmos para resolver y analizar problemas sobre el flujo de fluidos computacionales (Cruz et al., 2020). Ansys Fluent, dentro de su plataforma, reúne diferentes sistemas para física, mecánica de los sólidos, dinámica de los fluidos, electromagnetismos, parte nodal para análisis vibratorios, entre otros módulos importantes de análisis. Actualmente este software es una de las plataformas más desarrolladas en el mundo que arrojan resultados óptimos, porque utiliza entornos en tercera dimensión, lo cual permite visualizar los fenómenos físicos en su totalidad (Ansys, 2010). La dinámica de fluidos computacionales ha sido empleada a nivel nacional e internacional en una gran cantidad de campos de la ingeniería, por ser la ciencia en la simulación de flujo de fluido, transferencia de calor y fenómenos similares a estos, según diversas investigaciones relacionadas con CFD. Este análisis refuerza el conocimiento de los subprocesos subyacentes, lo que permite una mayor investigación y desarrollo de nuevas tecnologías, así como optimizar los sistemas existentes.

Sin embargo, la fiabilidad de los resultados obtenidos depende de la exactitud de la física, la química y de los modelos numéricos adoptados, así como de las condiciones de contorno considerado altamente confiable en la modelación y simulación computacional del lecho fluido (que puede ser difícil de obtener debido a inestabilidades en el "lecho de combustible sólidos"), la alimentación irregular de combustible, las fluctuaciones de presiones externas que afectan a la alimentación del aire, la formación de depósitos, la heterogeneidad de combustible, etc. (Ansys, 2013a). Es indispensable modificar la matriz energética renovable del Ecuador incrementando la participación de esta energía que representa actualmente un 6 \% (biomasa), para evitar las graves consecuencias medioambientales que ocasiona el uso exagerado de los combustibles fósiles y los efectos negativos de su explotación en la biodiversidad de los ecosistemas de la naturaleza (Yndurain, 2005). La Amazonía, por su cuenca hidrográfica del río Amazonas, constituye la mayor formación boscosa del mundo, y por su biodiversidad se considera la más rica en recursos genéticos. A partir de varios aportes se ha logrado conocer una parte de los potenciales energéticos en la provincia de Pastaza y la transición origina un incentivo al desarrollo de nuevas tecnologías energéticas sostenibles (Castro, 2011). Según consta en los estudios botánicos de las especies que pueblan la región amazónica, el bosque primario corresponde al $53.81 \%$ del total y de bosque secundario un 46.19 \% (Hetsch, 2004). La especie de interés como potencial de biomasa por su crecimiento rápido conocida como Piptcomoca discolor (J. E. González et al., 2018).

El estudio profundo del pigüe, como biomasa energética, por sus potenciales características físicas, químicas y térmicas, y por sus propiedades como combustible, ha permitido describir su comportamiento bajo un régimen de altas temperaturas, para el desempeño bajo las condiciones de una combustión completa, entre otros aspectos importantes (Zuñiga, 2018). Con base en el conocimiento establecido se pretende realizar el aprovechamiento completo del pigüe a escala industrial y su participación en la generación de energía como fuente renovable con todas las ventajas y bondades que brinda el uso de la biomasa como combustible (González et al., 2020). Por lo tanto, los resultados numéricos deben ser cuidadosamente evaluados y deben llevarse a cabo extensas validaciones experimentales. Junto con las mediciones experimentales, el modelado CFD es una herramienta eficiente de gran alcance en la investigación y el desarrollo 
de dispositivos de combustión, por esta razón, se ha ganado importancia en la investigación y desarrollo de hornos y calderas, en particular calderas de pequeña escala (Ansys, 2013a).

Uno de sus principales productos de simulación es el software Ansys Fluent de dinámica de fluidos computacional CFD-FLUENT. Este software es un código general multipropósito basado en el método de volumen y elemento finitos aplicado a una malla. La tecnología de FLUENT posee una paquetería completa para simulación, a partir de un módulo de dibujo en 3D, modelos matemáticos y esquemas de solución; un módulo para crear cualquier tipo de malla y un paquete de posprocesamiento (Ansys, 2010). Con el objetivo de evaluar, mediante la dinámica de fluidos computacionales, la influencia de diferentes parámetros de diseño y operación en la eficiencia de la combustión de Piptocoma discolor (pigüe) como combustible biomásico renovable para la Amazonía ecuatoriana.

\section{Metodología}

\section{Área de estudio}

El estudio se desarrolló en los cuatro cantones de la provincia de Pastaza tal como se detalla a continuación.

Arajuno, Mera, Santa Clara y Pastaza cuentan con una altitud de 550 a 960 m.s.n.m. medida con apoyo de un instrumento para la medición cartográfica GPS, con una humedad relativa entre 80 y $90 \%$ y una temperatura promedio de $22^{\circ} \mathrm{C}$ (Quezada, 2013). Esta información permitió seleccionar las zonas de interés del bosque secundario para extraer las muestras y especies (González et al., 2020; Murillo, 2004), con base en la metodología publicada por el Ministerio del Ambiente del Ecuador \& Organización de las Naciones Unidas para la Alimentación y la Agricultura (2014) además de aplicar el muestreo sistemático de parcelas y árboles para extraer las muestras por cantones (Camacho \& Murillo, 2018).

\section{Método de muestreo}

El estudio experimental se hizo previa categorización de acuerdo con el diámetro altura pecho (DAP) de la especie del Piptocoma discolor (pigüe) de la siguiente manera: en la primera 10$15 \mathrm{~cm}$, en la segunda $16-21 \mathrm{~cm}$, en la tercera $22-27 \mathrm{~cm}$ y en la cuarta $28-33 \mathrm{~cm}$; 12 árboles por cantón, lo que da un subtotal de 48 árboles de los 600 árboles estudiados con características morfológicas semejantes para extraer la muestra de biomasa según la norma (TAPPI, 203m 58 se utilizó para la cuantificación de la cantidad de alfa celulosa obtenida del 2018). Mediante instrumentos especializados se obtuvo el peso de $3 \mathrm{~kg}$ por troza y $36 \mathrm{Kg} /$ cantón. De los cuatro cantones se obtuvo $144 \mathrm{~kg}$ de muestras para la determinación según la fórmula de FernándezPuratich \& Oliver-Villanueva (2014).

\section{Astillado}

El astillado fue de un espesor de $2 \mathrm{~cm}^{2}$ y largo de $2 \mathrm{~cm}^{2}, 5 \mathrm{~cm}^{2}$ y $8 \mathrm{~cm}^{2}$ (Solar, 2013) y para la combustión la astilla contiene una humedad del 45 \% (Gobierno de Navarra, 2015). Se procedió al proceso de secado en un horno industrial American Range, a una temperatura automatizada con $\pm 5^{\circ} \mathrm{C}$. Asimismo, se utilizaron las normas COPANT-maderas 458 para las muestras y determinación del porcentaje de humedad y otros análisis físicos. 


\section{Estructura química}

Para la determinación de la composición química fue importante realizar la molienda para reducir el tamaño de la partícula según las recomendaciones de Solar (2013), la norma internacional UNE-EN-15149-2 (2011) y Castelló et al. (2017). La clasificación de la partícula del biocombustibles sólidos se hizo en 8 tamices, con luz de malla, $3000 \mu \mathrm{m} 850 \mu \mathrm{m}, 425 \mu \mathrm{m}, 300 \mu \mathrm{m}, 212 \mu \mathrm{m}$, $180 \mu \mathrm{m}, 150 \mu \mathrm{m}, 75 \mu \mathrm{m}$ y pulverizado en esta experimentación tal como manifiesta (Nogues et al., 2010). Al ser pesados y valorados el tamaño de partícula según la cantidad retenida en cada uno de los tamices de acuerdo a luz de malla para determinar los usos industriales como pellets, según (Rezaei et al., 2016) los porcentajes obtenidos.

Tabla 1. Datos termoquímicos para simulación en el software Ansys Fluent

\begin{tabular}{|c|c|}
\hline Análisis elemental & (\% peso en seco) \\
\hline Carbono & 46.03 \\
\hline Hidrógeno & 4.33 \\
\hline Nitrógeno & 0.02 \\
\hline Oxígeno & 30.58 \\
\hline Análisis proximal & (\% peso en seco) \\
\hline Material volátil & 73.04 \\
\hline Carbono fijo & 14.39 \\
\hline Cenizas & 0.57 \\
\hline Humedad & 12 \\
\hline Composición química & (\% peso en seco) \\
\hline Celulosa & 42.25 \\
\hline Hemicelulosa & 22.03 \\
\hline Lignina & 22.95 \\
\hline Análisis calorimétrico & 12.77 \\
\hline Poder calorífico & (MJ/kg) \\
\hline
\end{tabular}

Fuente: (González et al., 2019).

\section{Experimentación con software Ansys Fluent para la combustión}

\section{Tratamiento DE LOS DATOS}

Se realizó un análisis profundo basado en las expresiones cinéticas, de balances de masa, energía y cantidad de movimiento, de gases y sólidos, en estado estacionario y con todos los elementos térmicos y químicos. Se puede desarrollar varios modelos matemáticos de acuerdo con lo que el usuario desea analizar dentro del campo de la combustión. En este caso particular, 
el método de transporte de especies figura como el más adecuado para este análisis (Ansys, 2010) debido a que considera modelos matemáticos de la combustión.

Figura 1. Métodos y modelos matemáticos para sistemas de análisis de combustión (Zuñiga, E, 2018)

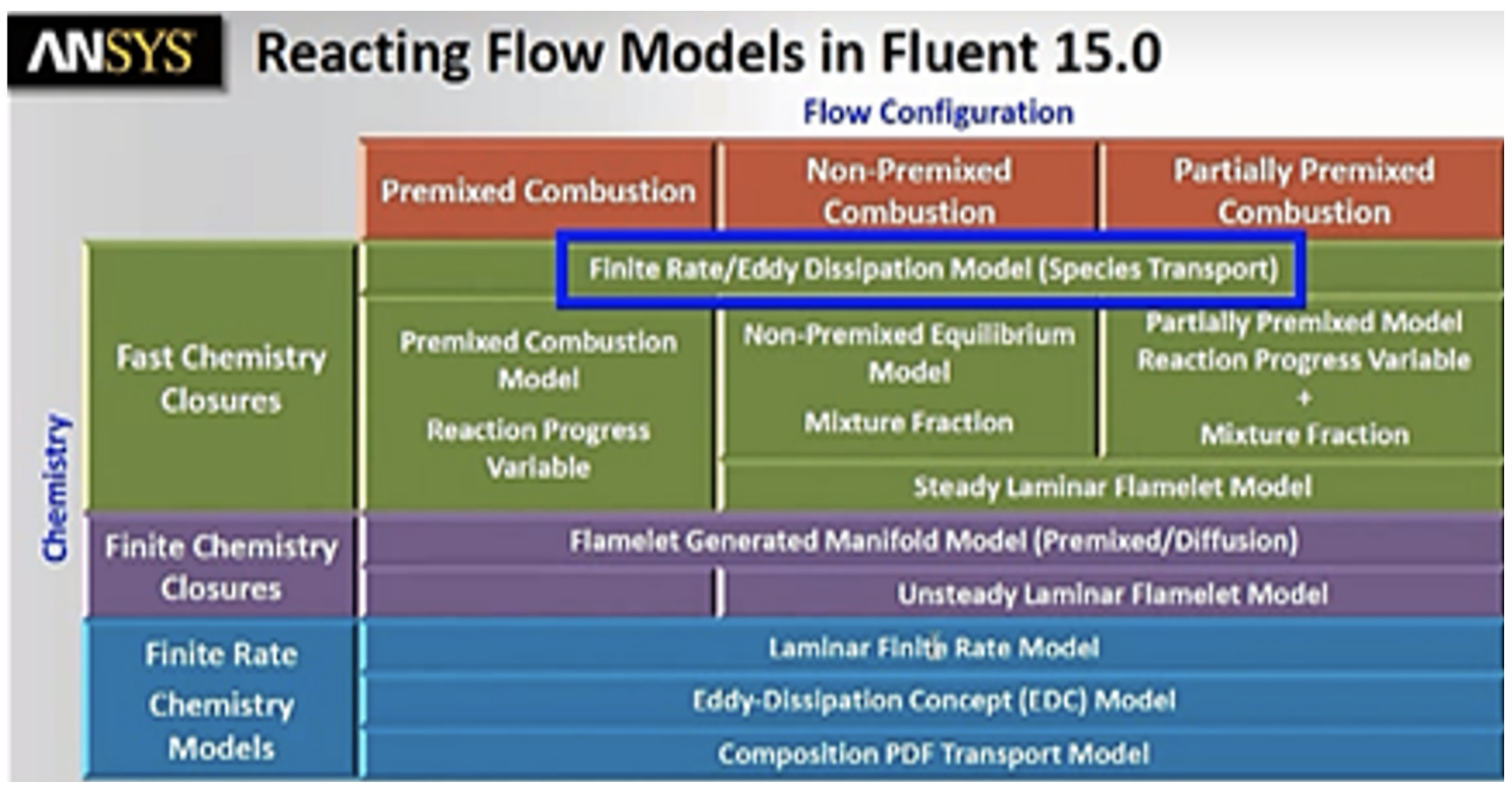

La figura 1 muestra las tres etapas que fueron desarrollados en el Ansys Fluent mediante la tasa finita/disipación de remolinos (transporte de especies) con las variables de progreso de la reacción del modelo de combustión premezclada, fracción de mezcla del modelo de equilibrio no premezclado y fracción de mezcla variable de progreso de reacción de modelo parcialmente premezclado permitió obtener los modelos matemáticos de la combustión.

\section{VARIABLES DEPENDIENTES E INDEPENDIENTES}

Se ha considerado en este trabajo el flujo de aire primario y el contenido de humedad de Piptocoma discolor, como también fueron necesarios los reaccionantes, calor especifico, diámetros promedios de los pellets y temperatura inicial en el horno. Las variables de respuesta fueron los siguientes parámetros o componentes de la combustión: cantidad de $\mathrm{N}_{2^{\prime}} \mathrm{O}_{2^{\prime}} \mathrm{CO}_{2^{\prime}} \mathrm{CO}$, $\mathrm{H}$, presión, temperatura, humedad, energía total y tiempo de quemado de las partículas de biomasa. El flujo de aire primario se presenta en cantidades menores en la parte baja del horno de análisis en un total de 84 orificios por donde ingresará aire y a su vez caerá la biomasa en forma de cenizas. Así también, las compuertas de abastecimiento de biomasa como de mantenimiento se convierten en un ingreso adicional de aire primario (Porteiro et al., 2009).

Este trabajo se desarrolló en una caldera de pellets cuyas medidas y configuración geométrica se presenta en la figura 2, lo cual no será motivo de análisis en esta investigación, así como los principios fundamentales de CFD, puesto que están bien documentados respectivamente. Se presenta una simulación completa de una caldera de pellets, que se basa en un código CFD disponible en el mercado, y el modelo de cama, que se basa en un reactor agitado perfecto sustentado internacionalmente (Porteiro et al., 2009). 
Figura 2. Isometría del volumen interno de la caldera empleada en este trabajo

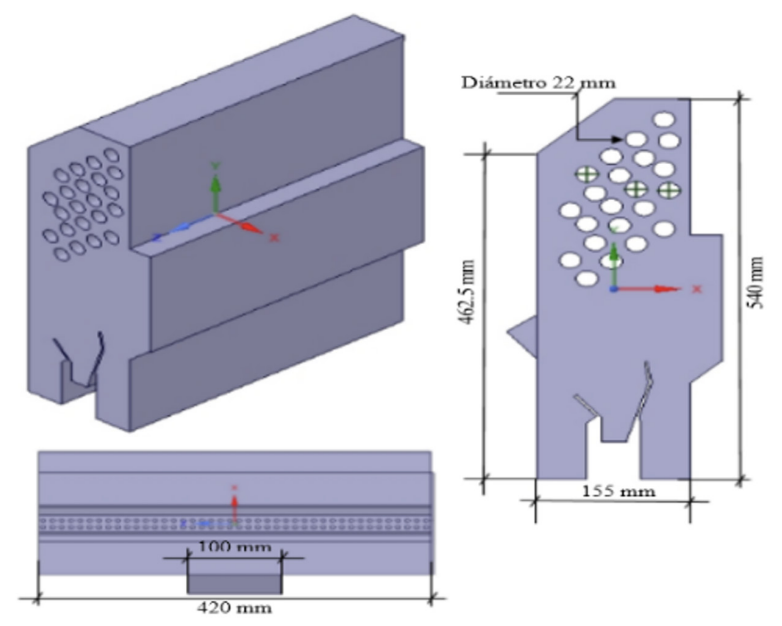

\section{Modelo matemático}

Las dinámicas de fluidos computacionales ocupan ecuaciones que representan un proceso de equilibrio para las especies de masa, cantidad de movimiento, energía y productos químicos. Se debe tener muy en cuenta la física de la partícula en las diferentes cantidades en las ecuaciones de balance (Ansys, 2013 ${ }^{\mathrm{a}}$ ).

Para este estudio se consideró el modelo matemático basado en el transporte de especies, en estudio de una especie particular amazónica, la cual con una previa caracterización facilita la configuración de la simulación pretendida.

\section{TRANSPORTE DE ESPECIES Y REACCIÓN}

$\frac{\partial}{\partial t}\left(\rho Y_{i}\right)+\frac{\partial}{\partial X_{i}}\left(\rho u_{i} Y_{i}\right)=\frac{\partial}{\partial X_{i}}\left[\rho Y_{i} \frac{\partial Y_{i}}{\partial X_{i}}\right]+R_{i}+S_{i}$

Donde:

$Y i=$ Fracción de masa de especies químicas

$\mathrm{ui}=$ Velocidad

$\mathrm{Ri}=$ Fuente de reacción

$\mathrm{Si}=$ Todas las demás fuentes

\section{ECUACIÓN DE CONTINUIDAD}

$\frac{\partial \rho}{\partial t}=\bar{v} \cdot(\overrightarrow{v \rho)}=0$

\section{Conservación DE MASA}

$\frac{-}{t}+\frac{(u)}{x}+\frac{(v)}{y}+\frac{(w)}{z}=0$ 


\section{ECUACIÓN DE CANTIDAD DE MOVIMIENTO}

La ecuación rige la dinámica de los fluidos derivada de la segunda ley de Newton: esta variación de la cantidad de movimiento de un objeto responde a las fuerzas que actúan sobre él. Aplicando este principio físico al volumen de control, y teniendo en cuenta el producto, se calculó la cantidad de movimiento por unidad de volumen de la partícula fluida.

\section{Cantidad de movimiento (momentum)}

$M x \frac{u}{t}+u \frac{(u)}{x}+v \frac{(u)}{y}+w \frac{(u)}{z}=-\frac{1}{(x)}+v \frac{{ }^{2}(u)}{x^{2}}+v \frac{{ }^{2}(u)}{y^{2}}+v \frac{{ }^{2}(u)}{z^{2}}$

My $\quad \frac{v}{t}+u \frac{(v)}{x}+v \frac{(v)}{y}+w \frac{(v)}{z}=-\frac{1}{(y)}+v \frac{{ }^{2}(v)}{x^{2}}+v \frac{{ }^{2}(v)}{y^{2}}+v \frac{{ }^{2}(v)}{z^{2}}$

$M Z \frac{w}{t}+u \frac{(w)}{x}+v \frac{(w)}{y}+w \frac{(w)}{z}=-\frac{1}{(z)}+v \frac{{ }^{2}(w)}{x^{2}}+v \frac{{ }^{2}(w)}{y^{2}}+v \frac{{ }^{2}(w)}{z^{2}}$

\section{BaLANCE de energía}

$\rho \frac{D i}{D t}=-p \bar{v} \cdot \vec{v}+\square \cdot \square \times(k \square \cdot T)+\Phi+S_{i}$

$\frac{E}{t}+u \frac{E}{x}+V \frac{E}{y}+w \frac{E}{z}=\frac{\lambda}{C_{p}} \frac{{ }^{2} E}{x^{2}}+\frac{\lambda}{C_{p}} \frac{{ }^{2} E}{y^{2}}+\frac{\lambda}{C_{p}} \frac{{ }^{2} E}{z^{2}}$

Aceleración local + tres términos de advección = difusión

Donde:

$x, y, z=$ son los ejes del sistema coordenado

$u, v, w=$ son los componentes radial, tangencial y vertical de la velocidad

$=$ densidad del fluido

$\mathrm{P}=$ presión absoluta

$\mathrm{E}=$ cantidad de calor del fluido

$\mathrm{Cp}=$ calor específico del fluido

$\lambda=$ conductividad térmica

$v=$ viscosidad cinemática del fluido

Considerando un flujo incompresible y de viscosidad constante (características de un fluido como el agua), el desarrollo de las ecuaciones de momentum para un flujo tridimensional y tridireccional es y. En el caso de encontrarse en presencia de un campo gravitatorio, la ecuación (9) ha de ser modificada, de acuerdo con la ecuación (4), sumándole al gradiente de la presión un término correspondiente a la fuerza que ejerce la gravedad sobre cada elemento de volumen, así como se presenta a continuación.

$$
\begin{aligned}
& \left(\frac{u}{t}+u \frac{u}{x}+v \frac{u}{y}+w \frac{u}{z}\right)=-\frac{p}{x}+g_{x}+\mu\left(\frac{{ }^{2} u}{x^{2}}+\frac{{ }^{2} u}{y^{2}}+\frac{{ }^{2} u}{z^{2}}\right) \\
& \left(\frac{v}{t}+u \frac{v}{x}+v \frac{v}{y}+w \frac{v}{z}\right)=-\frac{p}{y}+g_{y}+\mu\left(\frac{{ }^{2} v}{x^{2}}+\frac{{ }^{2} v}{y^{2}}+\frac{{ }^{2} v}{z^{2}}\right)
\end{aligned}
$$




$$
\left(\frac{w}{t}+u \frac{w}{x}+v \frac{w}{y}+w \frac{w}{z}\right)=-\frac{p}{z}+g_{z}+\mu\left(\frac{{ }^{2} w}{x^{2}}+\frac{{ }^{2} w}{y^{2}}+\frac{{ }^{2} w}{z^{2}}\right)
$$

La ecuación de continuidad para flujo incompresible se define como:

$$
\frac{u}{x}+\frac{v}{y}+\frac{w}{z}=0
$$

Donde al prescindir de los términos $\rho$ y $\boldsymbol{v}$, las ecuaciones tienen cuatro incógnitas $(\boldsymbol{u}, \boldsymbol{\mu}$, $w$ y p). Las ecuaciones de Navier-Stokes son ecuaciones diferenciales parciales acopladas y no lineales. A partir de estas ecuaciones se pueden obtener ecuaciones para casos especiales (flujo estacionario, bidimensional) omitiendo los términos adecuados (Hernández, 2014). Sin embargo, se describe las ecuaciones para su mejor comprensión.

$\rho \frac{D \overline{\mathrm{v}}}{D t}=\rho \breve{\mathrm{g}}-\overline{\mathrm{v}} \mathrm{p}+\mu \overline{\mathrm{v}}^{2} \overline{\mathrm{v}}+\frac{1}{3} \mu \overline{\mathrm{v}}(\overline{\mathrm{v}} \cdot \overline{\mathrm{v}})$

Excepto para flujos especiales y muy simplificados, las ecuaciones de Navier-Stokes se han resistido a ser solucionadas desde su desarrollo original. Actualmente, con el avance de las computadoras, se ha podido solucionar diversos fenómenos representados por estas ecuaciones. Aunque técnicamente son aproximadas, muchas de ellas han resultado ser válidas (Rubio et al., 2013).

\section{Proceso de LA dinÁMICA de FLUIDOS COMPUTACIONALES}

Se desarrolló la simulación en el Ansys Fluent siguiendo las siguientes etapas: a) geometría (selección, parámetros, dominio, forma y tamaño); b) malla (estructura métrica y calidad); c) física (propiedades de flujo, modelo matemático y condiciones de frontera), d) reporte (parámetros calculados, verificación y validación), y e) posprocesamiento (gráficos, vectores y trayectorias). Esta metodología fue considerada para evitar cualquier contratiempo en el proceso (Torres \& Naidiuk, 2018).

a. Modelo geométrico: es la herramienta de dibujo llamado 3D del Ansys-DesignModeler. A partir de esta geometría se logró importar cualquier módulo geométrico del sólido en $2 \mathrm{D}$ y $3 \mathrm{D}$. Sobre este proceso se obtiene la tecnología del mallado con el software Audesk Inventor (Autodesk, 2003).

b. Generación de malla: el mallado en Ansys-Meshing se desarrolló por la fragmentación del sólido en pequeños elementos llamados volúmenes finitos, los cuales pueden ser de tipo hexaédrico, prismático, tetraédrico y piramidal. Con la combinación de estas formas se puede llevar a cabo la fragmentación de cualquier geometría ajustando dichos elementos a la topología del dominio por modelar.

c. Física Set-Up en FLUENT: dentro de interfaz de Ansys Fluent, y siguiendo la física y la solución de las etapas de la simulación, se le conoce como Set-up al apartado donde se seleccionan los parámetros, ecuaciones, modelos de turbulencia y esquemas numéricos de solución para llevar a cabo la simulación. Al desarrollar los esquemas de solución (basado en presión y densidad), estado del flujo (estacionario o transitorio), modelos de turbulencia ( $\kappa^{-} \varepsilon, \kappa^{-} \omega$, Reynolds Stress, etc.), esquemas de solución (¡PRESTO!, SIMPLE, SIMPLEC, etc.), grado de solución (1er orden, $2^{\text {do }}$ orden, implícito, ex- 
plícito, etc.), entre otros. Además, dentro de este apartado, se seleccionan los pasos de tiempo (time-steps) en los que se requiere llevar a cabo la solución, el número de iteraciones, etc. Esto según el fenómeno de transporte de la especie que se tenga, el poder computacional disponible y el grado de exactitud deseado para obtener la composición de combustible.

d. Condiciones de frontera: Ansys Fluent y las condiciones de frontera de entradas, salidas, paredes y orificios son establecidas en el dominio por simular, según la zona de la geometría. Las combinaciones y la coherencia del modelo geométrico, dentro de la etapa de selección de las físicas, que se asignaron valores de las condiciones iniciales de todas aquellas fronteras correspondientes, analizados con todos estos parámetros de simulación de combustión de la especie Piptocoma discolor y se obtuvo las condiciones de contorno (Porteiro et al., 2009). Al realizar las reacciones de la combustión según la granulometría de partícula de la especie y las propiedades térmicas y químicas, de acuerdo con los datos obtenidos de la investigación de la especie y su configuración granulométrica.

e. Reporte del monitoreo de la simulación: el comportamiento de los resultados en cada interacción, para identificar la solución con tendencia a converger o a divergir, muestran los valores residuales de cada término de las ecuaciones por resolver y los resultados que arroja el software, para identificar las anomalías y obtener la solución de la convergencia satisfactoria, de acuerdo con la presión generada en lecho (Ansys, 2013b). Al aumentar la temperatura del gas, se mueven rápidamente por el número de choques contra las paredes, al aumentar la presión del recipiente de la pared fija y su volumen no puede cambiar, ley de Gay-Lussac (García, 1995).

f. Reporte de la combustión:Al aplicar altas temperaturas $723.65^{\circ} \mathrm{C}$ para la oxidación completa de la biomasa por el oxígeno del aire. En esta reacción se libera agua, gas cenizas y calor que alcanzan valores superiores a $1700^{\circ} \mathrm{C}$. El fuego-calor se propaga por los tres medios o por distintas combinaciones de transferencia de calor, entre ellos constan los siguientes: radiación, convección y conducción.

g. Posprocesamiento: finalmente, a través de Ansys y su módulo de post-procesamiento llamado ANSYS-Results, se obtuvo las variables de respuesta en FLUENT. Los datos fueron analizados y visualizados dentro de ANSYS-Results para interpretarlos mediante distintos gráficos, lo cual facilitó la comprensión del comportamiento de los campos de la velocidad, la turbulencia, la presión, etc. permitiendo obtener asimismo, los archivos y resultados arrojados por FLUENT (Ansys, 2013b).

\section{Resultados y discusión}

\section{Muestreo}

La estimación de las muestras obtenidas de los diámetros o categorías DAP de la especies fue establecida por González et al. (2021). De los cuatro cantones provincia de la Pastaza, representa una cantidad de árboles promedio de 1274 árboles/ha anualmente; que fue obtenido directamente y con un peso promedio total 419,26 ton/ha año. Del método indirecto de estimación promedio que cuenta de 0,47 tms/ha año (Pérez, 2004). En relación con el bosque secundario 
el Pigüe brinda una mejor alternativa de producción por su crecimiento rápido para su aprovechamiento, obteniendo 32`181.341,47 millones de toneladas métricas anuales de las 100987 ha. (J. González et al., 2018). Para la combustión a partir del secado, molienda y obteniendo 8 categorías de diferentes tamaños de granulometría.

\section{Granulometría y estructura química}

De acuerdo con el tamaño de la astilla efectuada, se procedió al proceso de triturado para obtener una granulometría (min. Diámetro (m) 0.000692; max. Diámetro (m) 0.000693), según la configuración y la reacción de la combustión para el Piptocoma discolor (González-Rivera et al., 2018).

En base a la tabla 1, de los procesos termoquímicos son válidos para su aprovechamiento energético, con propiedades térmicas similares a las de otras especies para biocombustibles y que se aprecian por las diferencias en su estructura (Urien, 2013). En esta especie por su regeneración, crecimiento rápido y por su composición química diferente.

\section{ANÁLISIS INMEDIATO Y PROXIMAL}

Con base en los resultados reflejados en la tabla 1, de la conversión de la biomasa en relación con volátiles, carbono, cenizas y su poder calorífico superior se muestra las diferencias de los residuos forestales (Lima, 2013) y de especies forestales (Portero, 2018).

De los resultados de análisis térmicos y químicos, de acuerdo con la tabla 1, influye el poder calorífico superior directamente en su eficiencia energética (Parikh et al., 2005). a diferencias con otras biomasa similares reportados (Portero., 2018), esto permitirá la producción, manejo y aprovechamiento de la biomasa, así como sucede con otras plantaciones que permiten satisfacer la demanda energética (Vignote, 2016). A continuación, en la figura 3, se evidencia las curvas (TDC) de la codigestión-aislados CHNS.

Figura 3. Curvas (TCD) de la codigestión-aislados CHNS

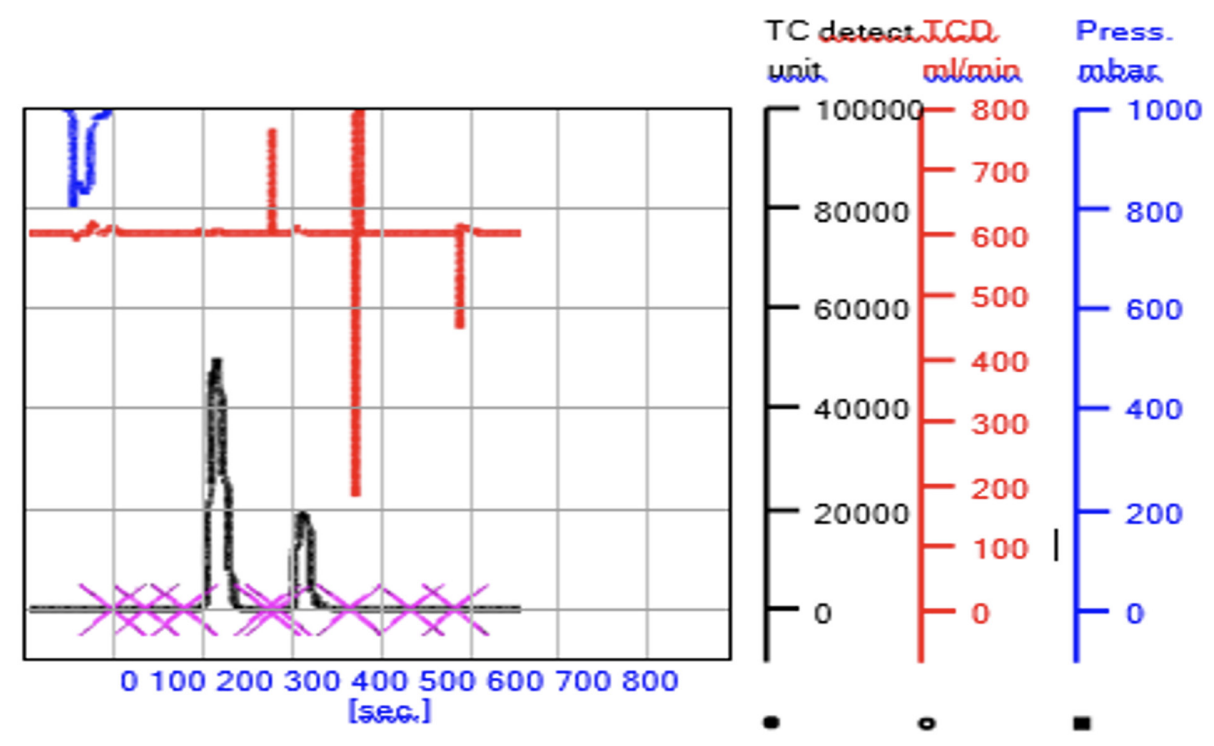


El análisis elemental se llevó a cabo con las diferentes técnicas de aplicaciones antes señaladas. Al comparar estos análisis con su propia especie, su composición varía las curvas de CHNS dentro de la misma especie, tal como se demuestra en la figura 3, debido a su hábitat, edad, suelo y ubicación en el árbol (tronco, ramas y raíces), por esas diferencias de la biodiversidad natural (Miranda \& Pereira, 2002).

Según los análisis de la tabla 1, los análisis de la composición elemental (\% ase seca) son similares; sin embargo, en condiciones amazónicas, el poder calorífico del Piptocoma discolor fue alto frente a otros materiales lignocelulósicos (Portero., 2018). La astilla de la especie en relación con el aserrín cuenta con la misma similitud (Abbas et al., 1994), como en otro estudio considerado por Abreu (2012).

\section{AnÁlisis y simulación de La combustión del Piptocoma discolor según el Ansys Fluent}

Según el análisis de la composición química y de sus propiedades térmicas de la especie, tanto en la modelación y simulación computacional como en la experimentación en una mufla, coincidieron los resultados obtenidos del máximo rendimiento, minimizando los problemas asociados ha dicho aprovechamiento. Las tecnologías de la combustión serán utilizadas, por sus principales ventajas de existencia en el país, asociadas a la disminución de emisiones contaminantes, también se considera la programación del software ANSYS FLUENT, un programa que valida muchas investigaciones prácticas a nivel mundial (Maldonado, 2018).

Se menciona que la oxidación del carbono implica la utilización de un exceso de aire y fue necesario otorgar cantidades mayores de oxígeno. Los parámetros o componentes de la combustión que fueron analizados son cantidad de $\mathrm{N}_{2}, \mathrm{O}_{2}, \mathrm{CO}_{2}, \mathrm{CO}, \mathrm{H}$, presión, temperatura, humedad, energía total y tiempo de quemado de las partículas de biomasa. Así también, se evaluó la efectividad de la simulación desarrollada mediante un análisis de convergencia matemática graficada.

Nitrógeno $\left(\mathbf{N}_{2}\right)$ : es un gas de combustión que abunda en estado sólido o líquido. Aunque este gas es un componente balastro y proviene del aire principal (figura 4, representada en color rojizo dentro de la gráfica de análisis), con un 76.41 \% N2 y corresponde a un porcentaje normal que se menciona en distintas literaturas que se enfocan en temas de la combustión (Canalís et al., 2014).

Figura 4. Fracción de masa del nitrógeno

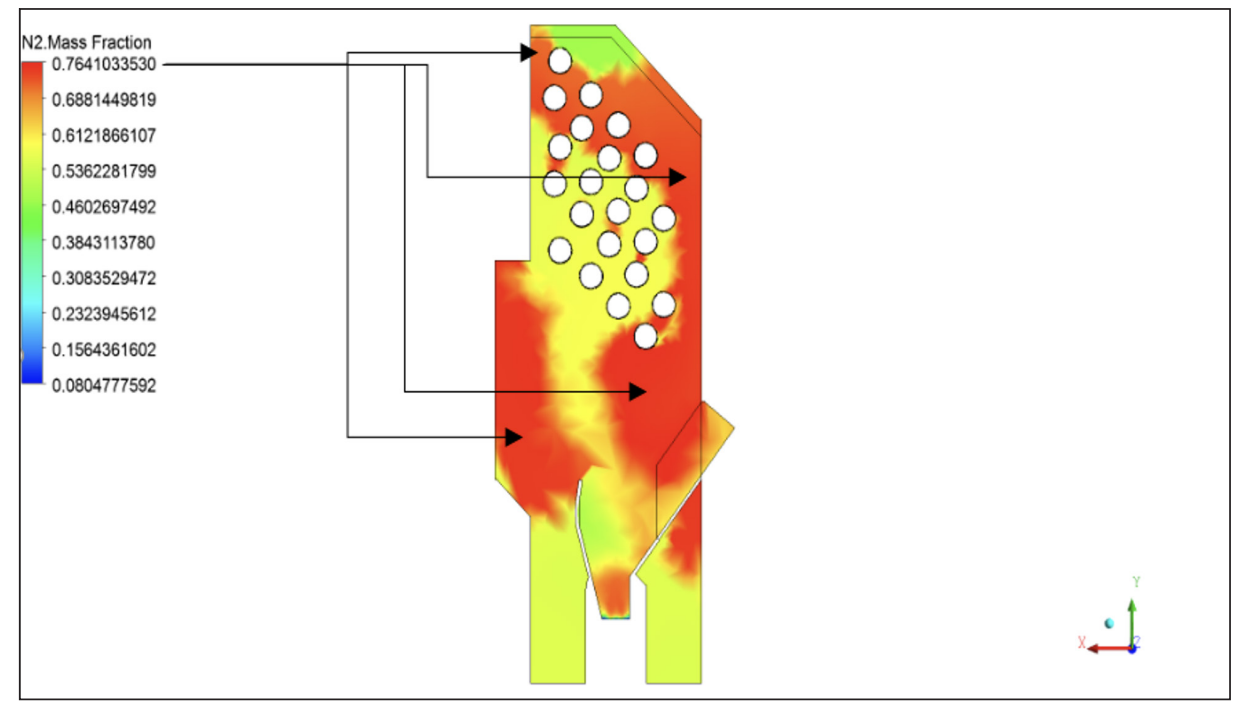


Oxígeno $\left(\mathrm{O}_{2}\right)$ : el valor fundamental de análisis en toda combustión tiene que ver con el oxígeno presente luego de la oxidación de la especie Piptocoma discolor, este, a su vez, proporciona resultados óptimos para analizar el rendimiento de la combustión. En este caso, en la figura 5 se muestra la cantidad de oxígeno que presenta luego de la convergencia a través del tiempo, un $22.34 \%$ en el ingreso principal de la caldera; mientras que en la cama del lecho su valor está entre el $2.4 \%$ y $4.97 \%$, lo que demuestra que las condiciones normales de operación están acordes con los valores típicos de los gases, líquidos y sólidos que bordean de entre el $2 \%$ y el 6 \% (CANALÍS et al., 2014).

Figura 5. Fracción de masa del oxígeno

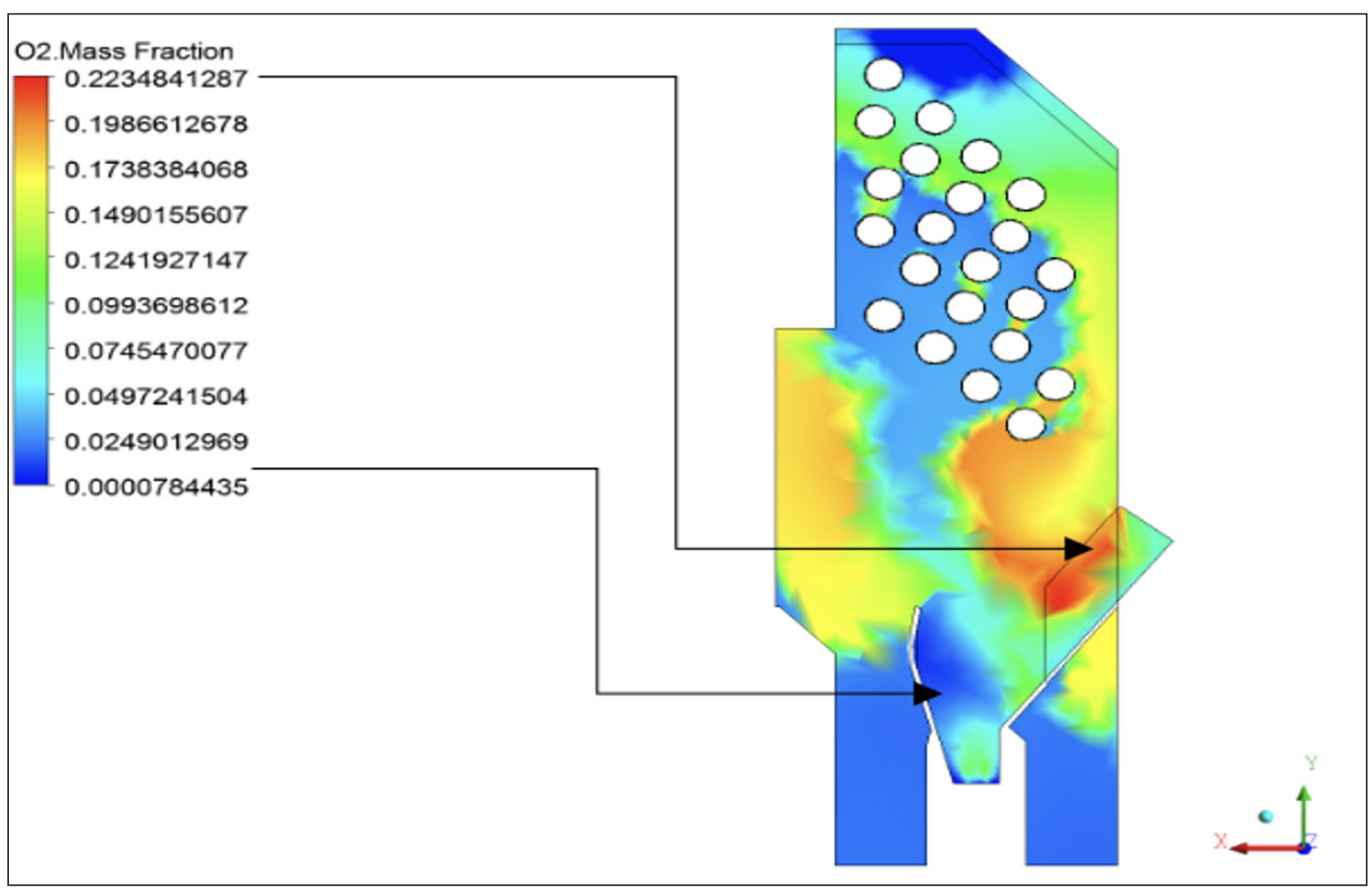

Dióxido de carbono $\left(\mathrm{CO}_{2}\right)$ : es un parámetro de análisis primordial para conocer el rendimiento de una combustión. Este tiene las siguientes características: es inodoro, incoloro, con un ligero gusto ácido (éste de acuerdo con el tipo de combustible). El resultado favorable es la producción de la mayor cantidad posible de $\mathrm{CO}_{2}$, puesto que representa una simulación de la combustión real del trabajo. Los valores obtenidos en cámaras de combustión y hornos de quemado oscilan del $12 \%$ al $14 \%$ como valores típicos. En la figura 6 se demuestra que la combustión del Piptocoma discolor no alcanzó a la producción $\mathrm{CO}_{2}$ del rango antes mencionado. En la simulación se obtuvo $8.25 \% \mathrm{CO}_{2}$ valor experimental diferente a otras especies (CANALís et al., 2014). 
Figura 6. Fracción de masa del dióxido de carbono

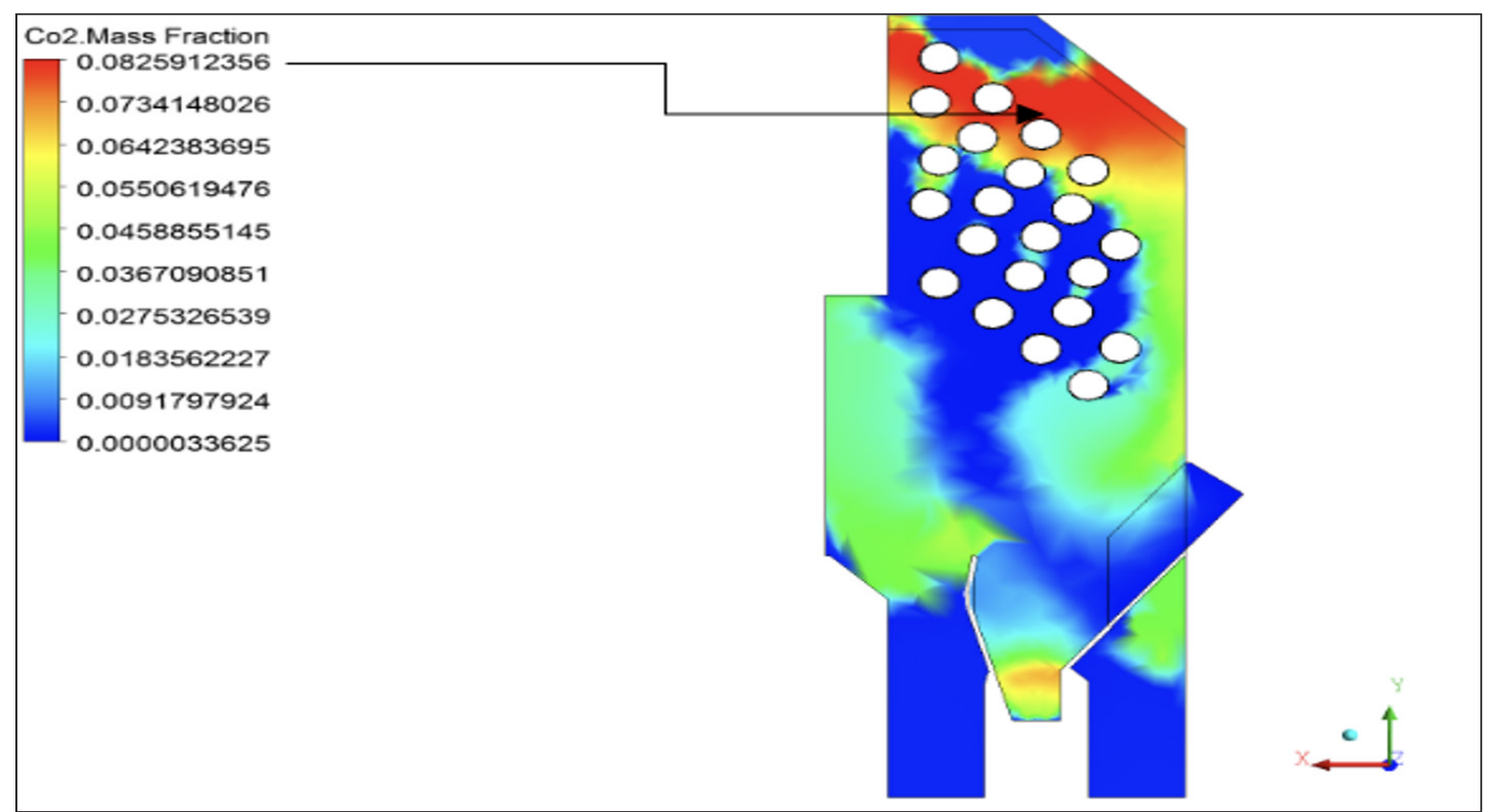

Monóxido de carbono (CO): lo que se busca en toda combustión es que este parámetro o componente se mantenga al mínimo posible para obtener un buen rendimiento en la combustión analizada. Lo ideal sería que este valor fuese cero y que se oxide todo el carbono, lo cual no sucede fácilmente. En la figura 7 se puede apreciar que la especie Piptocoma discolor, al ser combustionada, genera un mínimo de producción de CO, con un valor $4.04 \%$ que se desarrolla exclusivamente en la cama del lecho de la cámara, lo cual favorece rotundamente a no emitir elementos tóxicos en el ambiente (CANALís et al., 2014).

Figura 7. Fracción de masa de monóxido de carbono

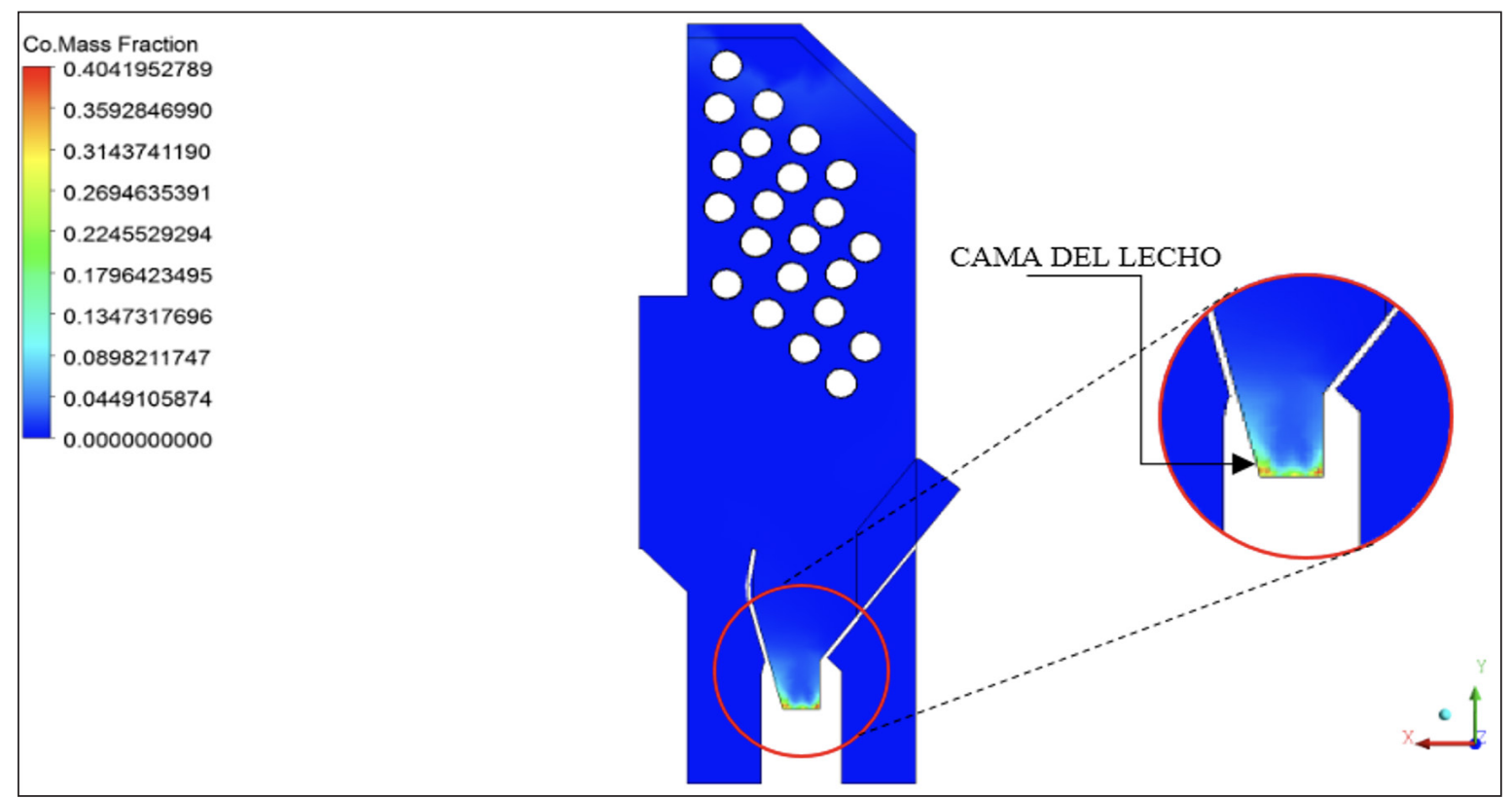


Hidrógeno $(\mathrm{H})$ : este un gas inodoro, incoloro e insípido con características diatómicas, que se disocia en átomos libres a altas temperaturas. En la figura 8 se constata que este gas, a medida que la temperatura aumentó, se desvolatizó notablemente a lo largo de todo el volumen analizado. Esto explica que la Piptocoma discolor, seca y sometida a altas temperaturas, genera un potencial energético satisfactorio (Saveris, 2014). Al ser alimentada con astillas en el reactor y combustionada a altas temperaturas, el rendimiento fue $71.46 \pm 10.58 \%$ y con los pellets de $66.12 \pm 12.76 \%$. Esto demuestra que la factibilidad de aprovechar astillas, serrín o pellets de esta especie es porque se consigue un menor flujo de alquitranes que pueden generar en las tuberías de las calderas (Torres et al., 2019).

Figura 8. Fracción de masa del hidrógeno

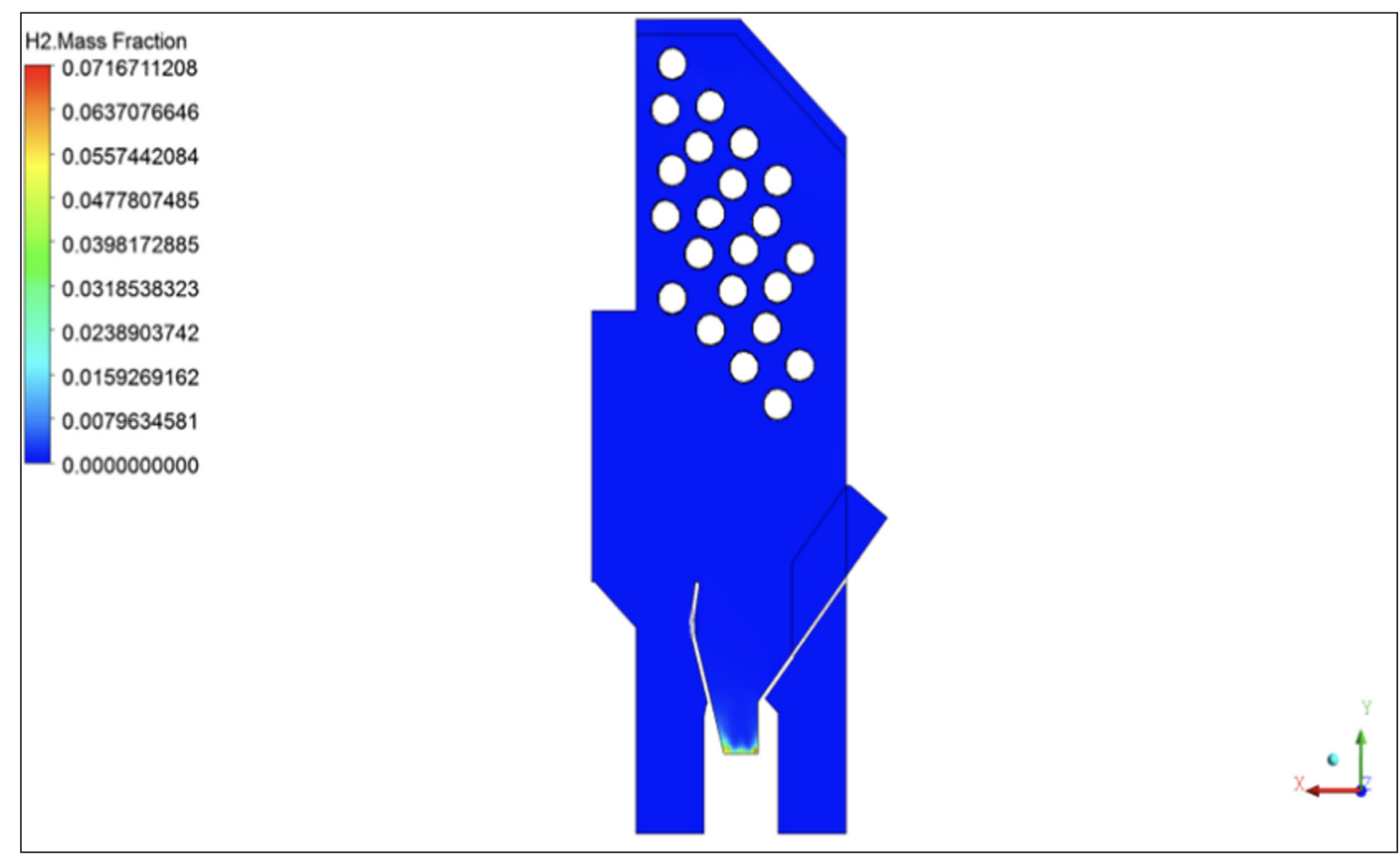

Presión y temperatura: los parámetros de presión y temperatura mostrados en las gráficas de la figura 9 conducen a pensar en la eficiencia del poder energético que posee la especie Piptocoma discolor; es decir, en condiciones de combustión, alcanzan valores considerables. En el caso de la temperatura, esta llega a un valor de $1773^{\circ} \mathrm{C}$. Esta permite transferir calor por convección mediante los tubos hacia el agua en su interior y logra fácilmente generar vapor saturado óptimo para utilizar en la industria productora de energía eléctrica a partir de turbinas de vapor (Spirax-Sarco, 2013). 
Figura 9. Presión y temperatura

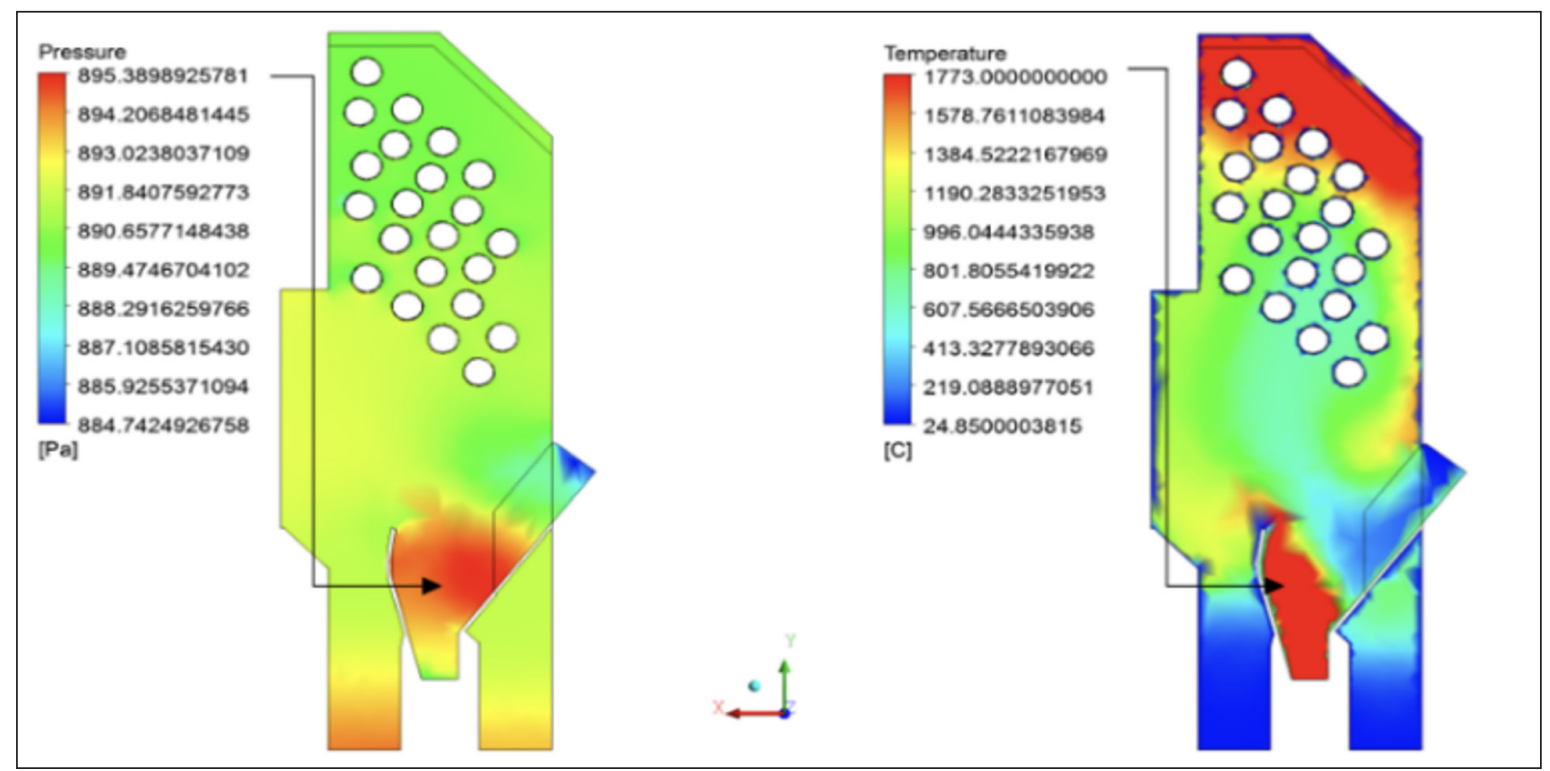

Humedad: aunque la mayor temperatura se verifica en la parte superior del horno, en esta zona se nota la humedad y agua volátil, la cual provocaría corrosión significativa en los tubos que transfieren calor. El porcentaje encontrado esta entre $7.06 \%$ a $9.89 \%$ de vapor de agua (figura 10).

Figura 10. Humedad presente en el interior de la caldera

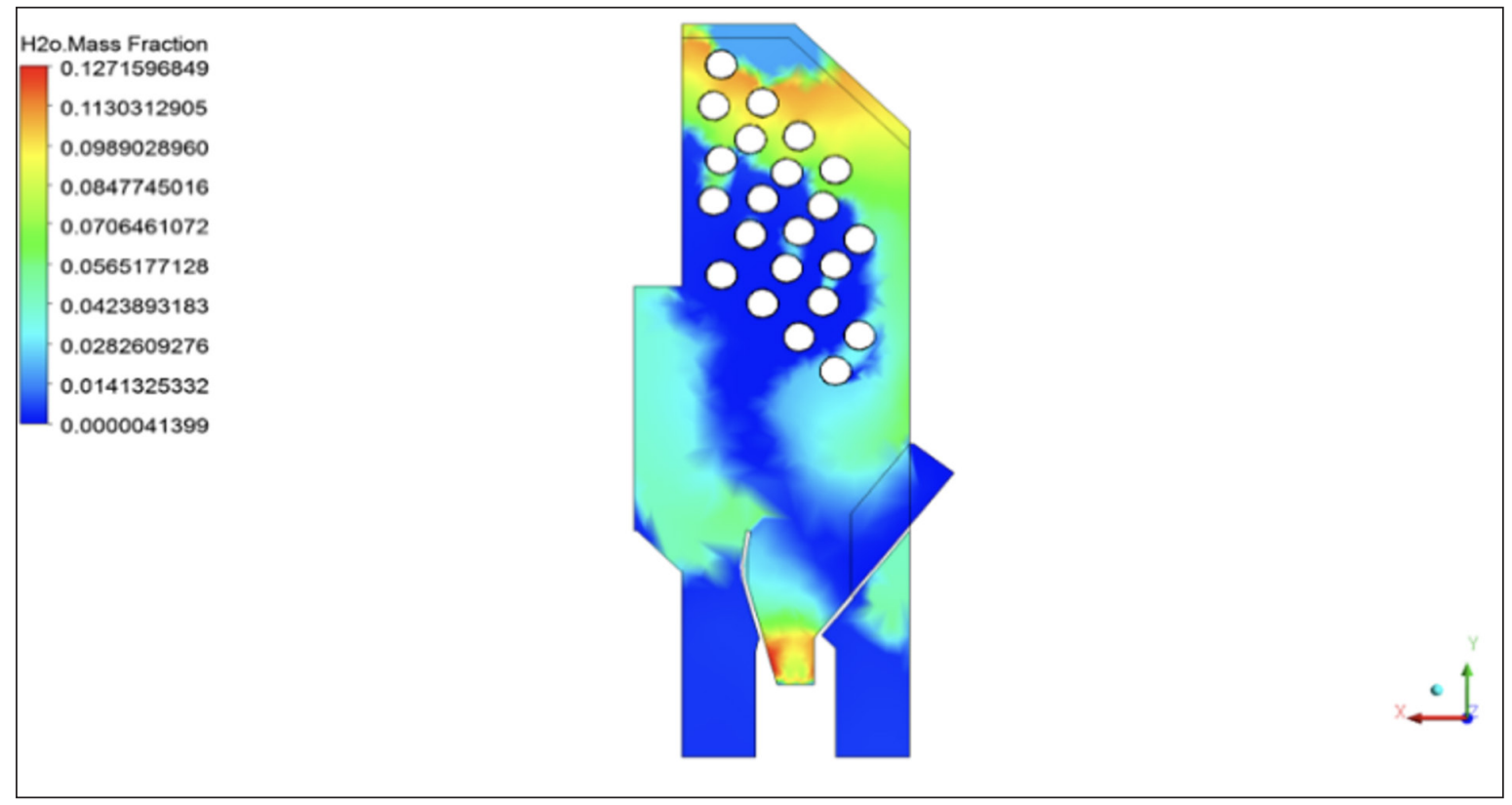

Energía total: se refiere a la cantidad de potencia energética requerida para quemar cantidades determinadas de biomasa. De acuerdo con la materia seca, se ve que la energía mayor requerida es $14490116 \mathrm{~J} / \mathrm{kg}$, es decir, en la cama del lecho, donde surge la quema de la biomasa, 
se necesita de un trabajo potencial de $14490116 \mathrm{~J}$, lo cual, traducido a Watts en un determinado tiempo, reporta un valor igual a 14490116 W; o lo que es lo mismo, 4 025.03 W.h, como consta en la figura 11. Esto concuerda con los estudios enfocados en biomasas (Abarca, 2000).

Figura 11. Energía total

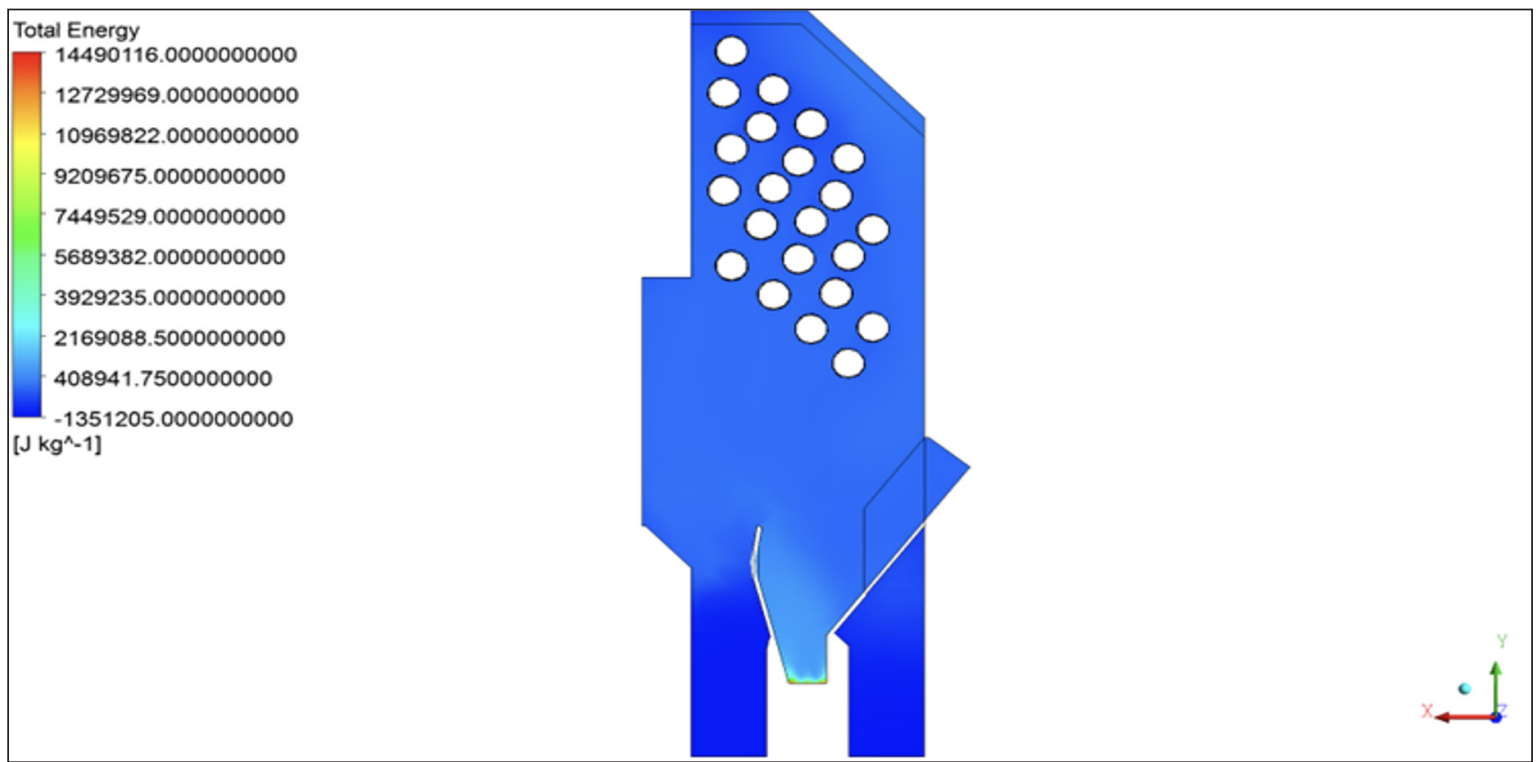

Tiempo de partícula: para lograr un completo quemado de cada una de las partículas de la biomasa, es necesario el paso del tiempo. Este tiempo de quema dependerá mucho del poder energético suministrado en la combustión. En la figura 12 se puede ver que el tiempo máximo de quemado es 2.16 segundos (Valencia, 2013).

Figura 12. Tiempo de la quema total de una partícula

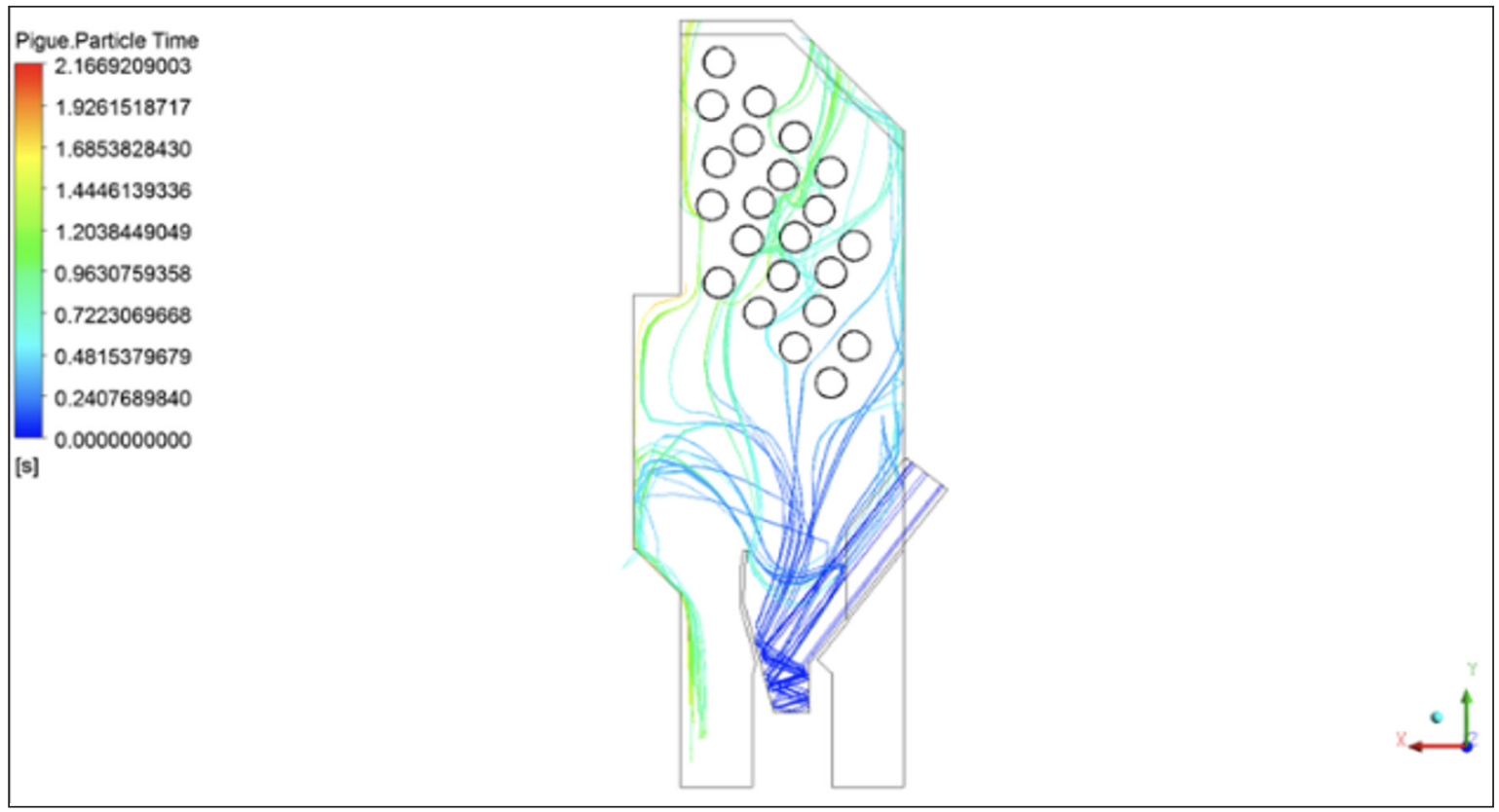


Convergencia (simulación efectiva): el Ansys Fluent, software especializado para sistemas de análisis, demuestra su efectividad a través de la convergencia desarrollada en sus gráficas de respuesta de ilustración final de la simulación total (figura 13). Aquí se explica que los algoritmos utilizados para la solución de la quema han sido los correctos y no existe desviación alguna en ninguno de los datos utilizados en este trabajo. Es decir, luego de que el software procesa los datos insertados en él, se grafican una serie de curvas de acuerdo con los parámetros estipulados en la solución matemática de la simulación, y estos deben llegar a converger para entender que no existen datos ficticios ni mal interpretados, conocidos como datos basura. Por lo tanto, se concluye que la simulación fue efectiva (Ansys, 2013b).

Figura 13. Ilustración final de la simulación total luego del procesamiento de datos

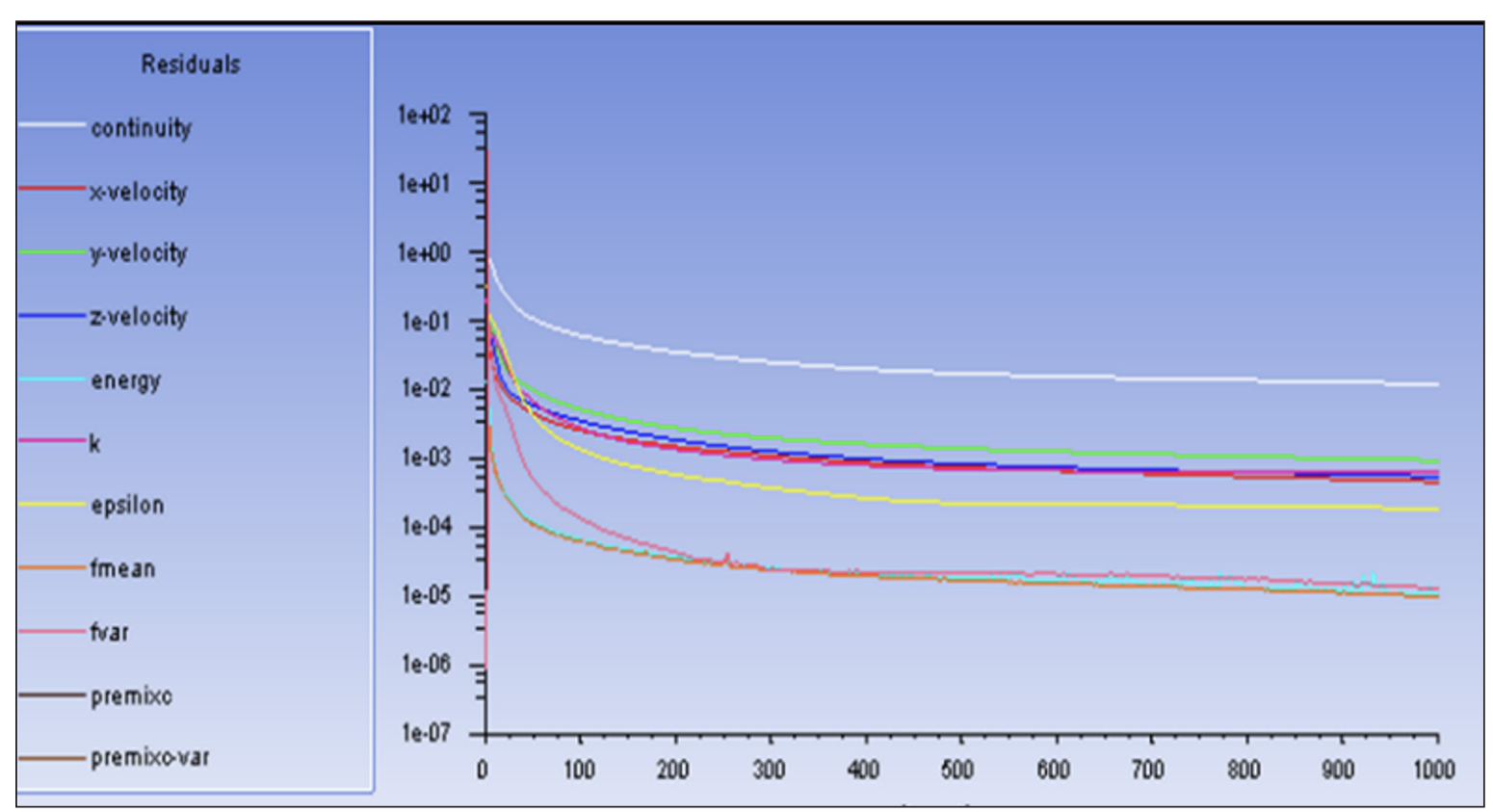

Al analizar el comportamiento térmico para la aplicación del sistema tecnológico, en la generación de energía eléctrica con ventajas para el sistema de combustión en calderas tipo parrillas y la disminución de emisiones de los gases de la combustión a menores temperaturas de $760^{\circ} \mathrm{C}$ a $871^{\circ} \mathrm{C}$, mientras que, en el sistema de horno en quema directa, conocidos como ciclos Rankine, su temperatura está por encima de los $1204^{\circ} \mathrm{C}$. Cuando se trata de madera o residuos forestales, las emisiones de $\mathrm{SO}_{2}$ son insignificantes y menos óxidos de nitrógeno (Nox). Al realizar la inversión con un costo menor su eficiencia del proceso de combustión, debe ser evaluada de acuerdo con la potencia necesaria de energía para su aplicación industrial (Loaiza, 2015).

El análisis de la simulación, a través del Ansys Fluent, en relación con la combustión de la biomasa, con una humedad de equilibrio del $12 \%$, demostró las siguientes relaciones: nitrógeno $76.41 \%\left(\mathrm{~N}_{2}\right)$, oxígeno $22.34 \%\left(\mathrm{O}_{2}\right)$, un valor de gas carbónico $8.25 \%\left(\mathrm{CO}_{2}\right)$, monóxido de carbono $4.04 \%$ (CO), hidrogeno $7.16 \%(\mathrm{H})$, con presión de $894(\mathrm{~Pa})$ y temperatura de $1773^{\circ} \mathrm{C}$, que permite generar vapor para producir energía eléctrica con una energía total requerida de $14490.12 \mathrm{KJ} /$ kg, como trabajo de $14490.12 \mathrm{KJ}$. A través de este programa computacional (Rosero, 2018) se ha logrado hacer la validación real que ocurre con esta herramienta de la dinámica de fluidos computacionales, similares a la experimental, a través de los análisis físicos, químicos y térmi- 
cos. Conociendo la información de las ecuaciones y aplicaciones de las energías renovables, los efectos positivos que muestra la especie es que no produce mucha ceniza ni afecta a la tubería de la caldera en la formación de incrustaciones, lo que la convierte en una alternativa para producir energía renovable sin daños al medio ambiente y equipos industriales.

\section{Conclusiones}

La provincia de Pastaza y la Amazonía ecuatoriana cuentan con un potencial productivo exuberante de esta biomasa forestal Piptocoma discolor que forma parte del bosque secundario, por ser una especie nativa y de crecimiento rápido con características fisicoquímicas y térmicas propicias para utilizarla como fuente de energía renovable.

La simulación dinámica de fluidos y los algoritmos de solución empleados demuestran que el método desarrollado de transporte de especies fue el correcto y la predicción del comportamiento de los gases como producto de la combustión de este fenómeno químico-exotérmico ocurrido en la validación del sistema.

La temperatura producida en la simulación del Piptocoma Discolor por efectos de la combustión ofrece valores altos de transferencia de calor para producir vapor saturado, el cual industrialmente se utilizaría como fluido propulsor para turbinas de vapor y transformaría la energía mecánica en eléctrica satisfactoriamente.

En esta simulación se puede constatar que la especie Piptocoma discolor emana poca toxicidad al ambiente y que el CO se mantiene al mínimo, que es lo que justamente se busca en una combustión de biomasas. Así, los gases del oxígeno y el hidrógeno muestran comportamientos satisfactorios, donde se ve que sus partículas se disocian notablemente.

\section{Referencias}

Abarca, P. (2000). Potencia y rendimiento de la caldera. ACHS. https://bit.ly/3H6cTC4

Abbas, T., Costen, P., Kandamby, N. H., Lockwood, F. C., \& Ou, J. J. (1994). The influence of burner injection mode on pulverized coal and biomass co-fired flames. Combustion and Flame, 99(3-4), 617-625. https://doi.org/10.1016/0010-2180(94)90055-8

Abreu, R. (2012). Utilización energética de la biomasa lignocelulósica obtenida de Dichrostachys cinera mediante procesos de termodescompocisión. En A. e. A (pp. 41-42). Universidad Politécnica delle Marche Dipartimmento di science Agrarie.

Ansys. (2010). Introduction to CFD Methodology. Ansys Fluent, 2, 17. https://bit.ly/3ve82LP

Ansys. (2013a). Guía de teoría fluido ANSYS. 15317 814. https://bit.ly/3vfhdfg

Ansys. (2013b). Guía teoría fluido ANSYS. https://bit.ly/3t1KZBf

Autodesk. (2003). Manual de usuario Autodesk (Copyright ed.).

Camacho, P., \& Murillo, O. (1997). Metodología para la evaluación de la calidad de plantaciones forestales recién establecidas. Agronomía Costarricense, 21(2), 189-205. https://bit.ly/355EwgO

Canalís, P., Royo, J., \& Sebastián, F. (2014). Co-combustión de carbón y biomasa. ROSAL MABRIK.

Castelló, M., Barrera, C., Pérez, É., \& Betoret, N. (2017). Reducción del tamaño de particulas y tamizado de sólidos. Universidad Politécnica de Valencia. https://bit.ly/3g305n7

Castro, M. (2011). Hacia una matriz energética diversificada en Ecuador. CEDA. http://biblioteca.olade. org/opac-tmpl/Documentos/cg00344.pdf

Cruz, Y., Valdés, P., Laffita, A., Gómez, M., \& Chuairey, C. (2020). Dinámica de fluido computacional: Revisión y análisis de las aplicaciones en la ingeniería. Revista Ciencias Técnicas Agropecuarias, 29(4), 93-104. https://bit.ly/3u3j1r9 
Fernández-Puratich, H., \& Oliver-Villanueva, J. V. (2014). Cuantificación de biomasa y valor energético de renovales de Quercus ilex en condiciones mediterráneas. BOSQUE (Valdivia), 35(1), 65-74. https://doi.org/10.4067/S0717-92002014000100007

García, M. (1995). La ley de Amontons y las indagaciones sobre el aire en la Academia de Ciencias de París (1699-1710), Asclepio, 47(1), 53-80. https://doi.org/10.3989/asclepio.1995.v47.i1.451

Gobierno de Navarra. (2015). Combustibles de biomasa: Tipos y caracteristicas. III Plan Energético de Navarra, horizonte 2020. Comisión Mixta de la Biomasa Forestal de Navarra. https://bit.ly/33YHx1l

González-Rivera, J., Jaramillo-Ponce, J., Pérez-Quintana, M., Sablón-Cossio, N., \& Oliva-Merencio, D. (2018). Evaluación físico-mecánicas de tableros a base del aserrín de pigüe (Piptocoma discolor) y bagazo de caña de azúcar en Pastaza. Revista Amazónica Ciencia y Tecnología, 7(2), 95-104. https://dialnet.unirioja.es/servlet/articulo?codigo=6977652

González, J., Coronel, B., Quevedo, V., Uvidia, H., Oliva, D., Morón, C., \& Campo, M. (2021). Biomass potential and kinetics of drying model of Piptocoma discolor (pigüe) as a source of renewable snergy source in Ecuador. Enfoque UTE, 12(1), 74-90. https://doi.org/10.29019/enfoqueute.695

González, J., Papue, A., González, V., Borja, A., \& Oliva, D. (2018). Crecimiento y conservación de Piptocoma discolor (pigüe) en la provincia de Pastaza, Ecuador. CFORES: Revista Cubana de Ciencias Forestales, 6(3), 366-379. https://bit.ly/300fOEE

González, J. E., Coronel, B., Quevedo, V., Uvidia, H., Oliva, D., Morón, C., \& Robles, M. (2020). Potencial de la biomasa y la cinética del modelo de secado de Piptocoma discolor (pigüe) como fuente de energía renovable en el Ecuador. Enfoque UTE, 12(1), 74-90. https://doi.org/https://doi. org/10.29019/enfoqueute.695

González, J. E., Morillo, C., García, J., Cárdenas, J., \& Oliva, D. (2019). Determinación del potencial energético del pigüe (Piptocoma discolor) en la Amazonía ecuatoriana. Ciencia Digital, 3(1), 78-97. https:// doi.org/10.33262/cienciadigital.v3i1.298

Hernández, A. (2014). Aplicación de un modelo turbulento bidimensional para la simulación de flujo a superficie libre en un canal horizontal [Tesis de posgrado, Universidad Nacional de Colombia]. Biblioteca VIRTUALPRO.

Hetsch, S. (2004). La comercialización de madera en la provincia de Pastaza. https://bit.ly/3G5qrMX

Lima, L. (2013). Evaluación de la composición química y propiedades físicas de madera y corteza de cuatro coníferas para la producción de bioenergía [Tesis de maestría, Universidad Autónoma de Nuevo León]. Repositorio académico digital.

Loaiza, M. (2015). Modelo de generación de energia a partir de biomasa forestal [Tesis de pregrado, Universidad de Chile]. Repositorio académico.

Ministerio del Ambiente del Ecuador \& Organización de las Naciones Unidas para la Alimentación y la Agricultura (2014). Evaluación nacional forestal: Resultados. FAO Ecuador. https://bit.ly/3H4Rucz

Maldonado, J. (2018). Analisis matemático y simulación númerica del comportamiento de fluidos en un caldero pirotubular vertical [Tesis de posgrado, Universidad Politécnica Salesiana]. Repositorio institucional.

Miranda, I., \& Pereira, H. (2002). The variation of chemical composition and oulping yield with age and growth factors in young Eucalyptus globulus. Wood and Fiber Scienc, 34(1), 140-145. https:// wfs.swst.org/index.php/wfs/article/view/49/49

Murillo, O. (2004). Evaluación de la calidad y estimación del valor en pie de la plantación forestal. Taller de Publicaciones del Instituto Tecnológico de Costa Rica.

Nogues, F., Galindo, D., \& Rezeau, A. (2010). Energía de la biomasa (Vol. 1). Prensas Universitarias de Zaragoza.

Parikh, J., Channiwala, S. A., \& Ghosal, G. K. (2005). A correlation for calculating HHV from proximate analysis of solid fuels. Fuel, 85(5), 487-494. https://doi.org/10.1016/j.fuel.2004.10.010

Pérez, P. (2004). Cuantificación de los recursos de biomasa forestales en la provincia de Soria y evaluación de alternativas para su aprovechamiento energético. Montes (75), 17-25. 
Porteiro, J., Collazo, J., Patiño, D., Granada, E., Moran, J., \& Míguez, J. (2009). Numerical modeling of a biomass pellet domestic boiler. Energy \& Fuels, 23(2), 1067-1075. https://doi.org/https://doi. org/10.1021/ef8008458

Portero, H. (2018). Estudio experimental de peletizado en planta piloto y de combustión en caldera de biomasa a baja potencia [Tesis doctoral, Universidad de Castilla-La Mancha]. Repositorio RUIdeRA.

Rezaei, H., Jim, C., Lau, A., \& Sokhansanj, S. (2016). Size, shape and flow characterization of ground wood chip and ground wood pellet particles. Podwer Technology, 301, 737-746. https://doi. org/10.1016/j.powtec.2016.07.016

Rosero, W. (2018). Utilización de dinámica de fluidos computacional CF-ANSYS fluent para la simulación de un gasificador [Tesis de grado, Universidad de Bogotá Jorge Tadeo Lozano]. Repositorio institucional.

Rubio, J. d. J., Ordaz, G., Jiménez, M., \& Cabrera, I. (2013). Solución general de la ecuación de Navier-Stokes para la dinámica de un fluido viscoso homogéneo en una tubería abierta. Revista Mexicana de Física, 59(3), 217-223.

Saveris, T. (2014). Distribución de vapor. testo Saveris 2. https://bit.ly/3LQPVBA

Solar, A. (2013). Metodología para la optimización del aprovechamiento energético de los recursos de biomasa: Aplicación a la comunidad valenciana [Tesis doctoral, Universidad Politécnica de Valencia]. Repositorio institucional.

Spirax-Sarco, S. A. (2013). Distribucion del vapor. TR-GCM-03. https://bit.ly/3vilUuyK

TAPPI. (2018). TAPPI standars: Regulations and style guidelines. https://bit.ly/3H4hFA4

Naidiuk, L., \& Torres, B. (2018). Introducción al análisis térmico y fluidos mediante Ansys. Abya-Yala.

Torres, C., Chaves, M., Urvina, L., \& Moya, R. (2019). Evaluación de la incidencia de pellets y astillas de madera en el desempeño de un gasificador tipo downdraft. Revista Forestal Mesoamericana Kurú, 15(1), 23-34. https://doi.org/10.18845/rfmk.v15i1.3847

Urien, A. (2013). Obtención de biocarbones y biocombustibles mediante pirolisis de biomasa residual [Tesis de maestría, Universidad Nacional de Educación a Distancia]. Digital.CSIC. https://bit. ly/3r3uB3z

Valencia, W. J. J. (2013). Modelamiento y simulación de la combustión de una partícula de carbón en un gas combustible. [Tesis de maestría, Universidad Nacional de Colombia]. Fdocuments.ec. https:// bit.ly/3KGiJMJ

Vignote, S. (2016). Procesos termoquímicos de la madera y en general de la biomasa. https://doi. org/10.13140/RG.2.2.21490.50889

Yndurain, F. (2005). Energía: Presente y futuro de las diversas tecnologías. Academia Europea de Ciencias y Artes. https://bit.ly/3u3w9wr

Zuñiga, E. (2018). Modelación y simulación de la combustión de Piptocoma discolor (pigüe) como fuente biomásica con el uso de fluidos dinámicos computacionales Universidad Estatal Amazónica]. Puyo. 